\begin{tabular}{|c|l|}
\hline Title & Electrochemical Formation of Size Controlled InP Nanostructures U sing A nodic and Cathodic Reactions \\
\hline Author(s) & Sato, Taketomo; Fujino, Toshiy uki; Hashizume, Tamotsu \\
\hline Citation & $\begin{array}{l}\text { Electrochemical and Solid-State Letters, 10(5), H153-H155 } \\
\text { https://doi.org/10.1149/1.2713662 }\end{array}$ \\
\hline Issue Date & 2007 \\
\hline Doc URL & http://hdl.handle.net/2115/20209 \\
\hline Type & article \\
\hline File Information & E\&S-SL10-5.pdf \\
\hline
\end{tabular}

Instructions for use 


\title{
Electrochemical Formation of Size-Controlled InP Nanostructures Using Anodic and Cathodic Reactions
}

\author{
Taketomo Sato, ${ }^{\mathrm{z}}$ Toshiyuki Fujino, and Tamotsu Hashizume
}

Research Center for Integrated Quantum Electronics, Hokkaido University, Sapporo 060-8628, Japan

\begin{abstract}
A two-step electrochemical process using anodic and cathodic reactions was developed to form size-controlled nanostructures on $\operatorname{InP}(001)$ substrates. After anodic formation of a nanopore array, the cathodic decomposition process was applied to reduce the thickness of InP nanowalls. The etching rate of the nanowalls was extremely small and strongly dependent on the cathodic bias and crystal orientations of the wall surface. Wall thickness could be controlled in the range of 10-30 nm by changing the cathodic bias and processing time.

(C) 2007 The Electrochemical Society. [DOI: 10.1149/1.2713662] All rights reserved.
\end{abstract}

Manuscript submitted November 9, 2006; revised manuscript received January 10, 2007. Available electronically March 13, 2007.

High-density formation of semiconductor nanostructures has recently been intensely researched for applications such as photonic crystals, quantum and optoelectronic devices, and chemical and biochemical sensors. Mainstream approaches to forming semiconductor nanostructures have used conventional methods such as lithography, dry etching, or crystal growth processes. For example, reactive ionbeam etching (RIBE), molecular beam epitaxy (MBE), and metallorganic vapor phase epitaxy (MOVPE) are common techniques for this purpose in the semiconductor community. One possible alternative approach is to apply the electrochemical process to semiconductors. With this process, a wide variety of semiconductor nanostructures can be obtained by modifying the surface using electrochemical anodic and cathodic reactions. The electrochemical process seems to be extremely promising for creating semiconductor nanostructures due to its unique features such as being a lowtemperature process, causing low processing damage, and having a simple process and a low cost.

The most well-known application of the electrochemical process is for forming a porous $\mathrm{Si}$ structure using the anodic reaction in an HF-based electrolyte. ${ }^{1-3}$ This has given a visible light-emitting property to Si by modifying the surface. Canham ${ }^{3}$ reported that the photoluminescence (PL) peak obtained from porous Si showed a significant blue shift with reference to the bandgap energy of a bulk Si, showing evidence of quantum confinement in porous structures. Several groups later reported on porous structures made of III-V semiconductor materials such as GaAs, ${ }^{4,5} \mathrm{GaP},{ }^{6,7} \mathrm{InP},{ }^{8-10}$ and GaN. ${ }^{11,12}$ Up to the present, various crystal orientations and electrochemical conditions have been investigated on III-V materials to reveal structural properties and their tunability. We have recently succeeded in the anodic formation of arrays of straight nanopores on n-InP(001) substrates. ${ }^{13-15}$ The straightness of pores in the depth direction has been dramatically improved using an HCl-based electrolyte containing a small amount of $\mathrm{HNO}_{3}$, resulting in the formation of a high-density array of InP nanowalls with a high aspect ratio. These kinds of unique nanostructures have not been obtainable by other methods.

In this article, we report size-controlled InP nanostructures formed by a two-step electrochemical process. This process consists of self-organized pore formation and the subsequent dissolution of pore walls caused by changing the polarity of semiconductor substrates in the same electrolyte. As for pore formation, we have already reported that structural features such as pore diameter and depth can be controlled by anodic conditions and the ion concentration of electrolytes. ${ }^{14,15}$ Hence, this article focuses on the cathodic decomposition process to achieve precise control of wall thickness in a nanometer-scale range.

The setup of the electrochemical process used in this study is shown in Fig. 1a. The process was performed using a standard cell with three electrodes, i.e., an n-type $\operatorname{InP}(001)$ electrode $(n=1$

${ }^{\text {z }}$ E-mail: taketomo@rciqe.hokudai.ac.jp $\times 10^{18} \mathrm{~cm}^{-3}$ ) as a working electrode (WE), a Pt counter electrode (CE), and a saturated calomel electrode (SCE) for a reference. The electrolyte consists of $1 \mathrm{M} \mathrm{HCl}(200 \mathrm{~mL})$ with $\mathrm{HNO}_{3}(3 \mathrm{~mL})$. To supply current, a $\mathrm{GeAu} / \mathrm{Ni}$-ohmic contact was made on the back side of the InP substrate using the conventional evaporation and annealing process. Figure $1 \mathrm{~b}$ shows the control sequence of the bias applied to the semiconductor electrode with reference to the SCE. The anodic bias was first applied to the semiconductor electrode to obtain high-density porous structures. In this study, the anodic bias, $V_{\mathrm{a}}$, and anodization time, $t_{\mathrm{a}}$, were set at $4 \mathrm{~V}$ and $60 \mathrm{~s}$. These conditions are among the most suitable for forming uniform and straight nanopore arrays. Figure 1c shows a typical scanning electron microscope (SEM) image of the porous structure formed by the anodization process. The uniform pores orderly aligned on the $\mathrm{n}-\mathrm{InP}(001)$ substrates and constructed a triangular lattice. These features are very similar to the $\mathrm{Al}_{2} \mathrm{O}_{3}$ porous structures reported by Masuda et al. ${ }^{16} \mathrm{We}$ have previously reported that the InP nanowalls were standing vertically to a (001) plane, keeping good uniformity over $19 \mu \mathrm{m} .{ }^{95}$ From the statistical analysis, the average pore size and wall thickness were estimated to be 103 and $28 \mathrm{~nm}$, respectively. In this study, the porous structure shown in Fig. 1c was used as a template for the subsequent cathodic process.

Next, the cathodic bias, $V_{\mathrm{c}}$, was applied to the sample in the same electrolyte to reduce wall thickness using the following cathodic reaction

$$
\mathrm{InP}+3 \mathrm{H}^{+}+3 \mathrm{e}^{-} \rightarrow \mathrm{In}+\mathrm{PH}_{3}
$$

The above reaction has been investigated by several groups ${ }^{17-19}$ for planar InP substrates. The equation might give the impression that metallic In remains on the InP surface, but this is not true when using an acid electrolyte with a low $\mathrm{pH}$ value below 1.0 , as in this case. Yao and Itaya ${ }^{18}$ and Seo et al. ${ }^{19}$ reported that the smooth and clean InP surface was obtained in the acid electrolyte where metallic In was easily transferred to the $\mathrm{In}^{+}$ion. In this study, we applied the cathodic decomposition process to porous structures for the first time. To clarify the tunability of the thickness of InP nanowalls, the basic properties were investigated on samples prepared by systematically changing the cathodic bias, $V_{\mathrm{c}}$, and processing time, $t_{\mathrm{c}}$. All electrochemical experiments were performed in the dark at room temperature.

Figures $2 \mathrm{a}$ and $\mathrm{b}$ show plan-view SEM images of the sample decomposed at $V_{\mathrm{c}}=0.75 \mathrm{~V}$ for $t_{\mathrm{c}}=40 \mathrm{~s}$ and the sample decomposed at $V_{\mathrm{c}}=0.75 \mathrm{~V}$ for $t_{\mathrm{c}}=80 \mathrm{~s}$. The thickness of the n-InP walls was found to decrease using the cathodic decomposition process. Average thicknesses of 22 and $17 \mathrm{~nm}$ were obtained for the sample shown in Fig. 2a and b, both of which are thinner than the initial value of $28 \mathrm{~nm}$ obtained for the porous sample just after the anodization shown in Fig. 1c. This indicates that the pore wall dissolved into the electrolyte during the cathodic reaction. By comparing the samples shown in Fig. 2a and b, the etching rate of the n-InP walls 


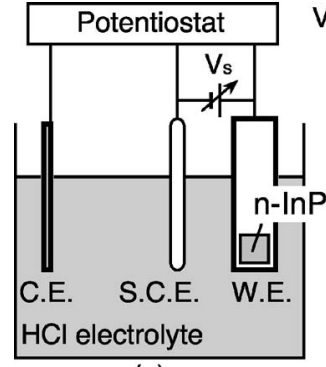

(a)

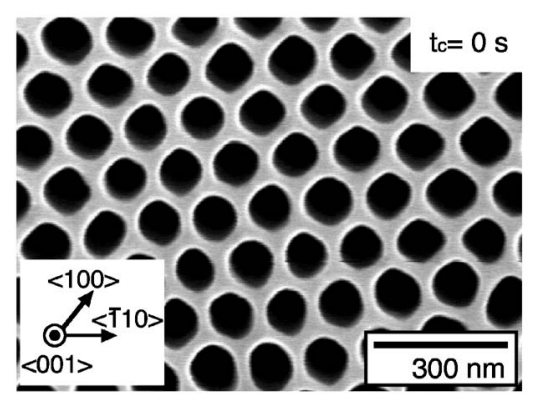

(c)

Figure 1. (Color online) (a) Setup of electrochemical process and (b) control sequence of bias applied to n-InP electrode. (c) Plan-view SEM image of template porous sample formed by anodization at $V_{\mathrm{a}}=4 \mathrm{~V}$ for $t_{\mathrm{c}}=60 \mathrm{~s}$.

was found to be strongly dependent on crystal orientation, where the $\{100\}$ planes appeared on the surface of InP walls as the processing time, $t_{\mathrm{c}}$, increased.

The average wall thickness, $d_{\mathrm{w}}$, measured on the samples after cathodic decomposition are plotted in Fig. 3 as a function of the processing time, $t_{\mathrm{c}}$. Three kinds of curves were drawn by changing the cathodic bias, $V_{\mathrm{c}}$, such as $-0.5,-0.75$, and $-1.0 \mathrm{~V}$. As seen in Fig. 3, the wall thickness thinned with increasing cathodic bias, $V_{\mathrm{c}}$, from -0.5 to $-1.0 \mathrm{~V}$. This is consistent with the basic idea that the etching rate is determined by the value of the cathodic currents contributing to the reaction described in Eq. 1. Wall thickness also decreased with processing time, but the behavior was slightly complicated. At the beginning of the cathodic decomposition process, wall thickness almost linearly decreased with a constant etching rate on the InP walls. Then, the slope angles gradually decreased and wall thickness was approaching the saturation values. These features are applicable for all three curves obtained at the different $V_{\mathrm{c}}$, as shown in Fig. 3.

The lateral etching rates of the InP walls were estimated to be approximately $0.3,0.2$, and $0.08 \mathrm{~nm} / \mathrm{s}$ for $V_{\mathrm{c}}=-0.5,-0.75$, and $-1.0 \mathrm{~V}$, respectively, in the initial linear region below $t_{\mathrm{c}}=40 \mathrm{~s}$. The values obtained here are extremely small, as compared with the conventional wet chemical etching of InP,${ }^{20}$ and show good tunability of the wall thickness in a nanometer-scale range. In the saturation region, the wall thickness is expected to be controllable in the selflimiting mode. For example, in the case of the samples decomposed at $V_{\mathrm{c}}=-1.0 \mathrm{~V}$, the difference in average wall thickness stayed within $5 \mathrm{~nm}$ in the saturation region from $t_{\mathrm{c}}=40-120 \mathrm{~s}$. From these results, the precise control of wall thickness was found to be achievable in the range of $d_{\mathrm{w}}=10-30 \mathrm{~nm}$ by changing the cathodic bias and decomposition time, as shown in Fig. 3.

One of the possible explanations for the time-dependent etching rate is as follows. At the beginning of the cathodic decomposition process, the InP nanowalls are uniformly etched in any lateral direction. In this stage, the wall thickness decreases almost linearly with the processing time, $t_{\mathrm{c}}$, where the etching rate is controlled by the value of cathodic currents. Then, the surface area of $\{100\}$ planes become larger in the pore walls because the etching rate in $\langle 100\rangle$

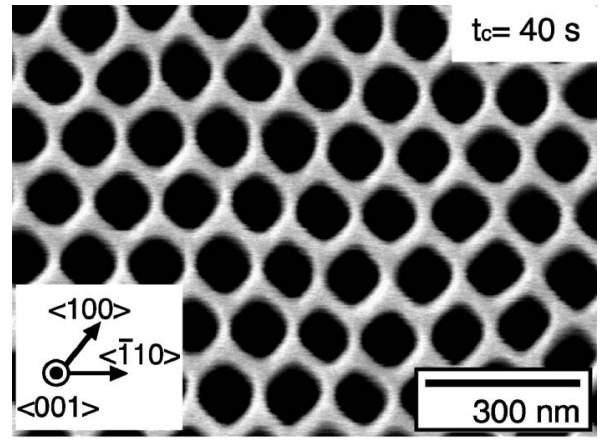

(a)

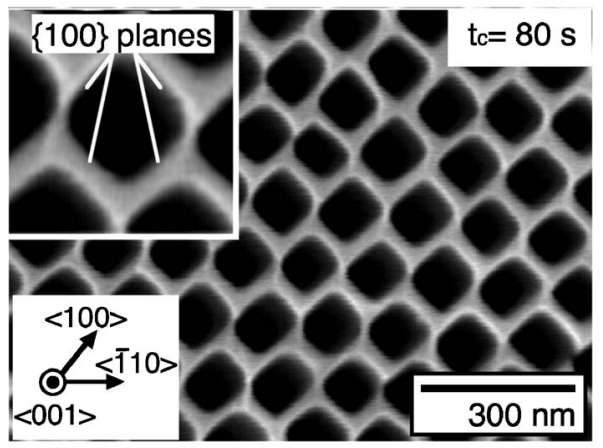

(b)

Figure 2. Plan-view SEM images of porous samples after cathodic decomposition at $V_{\mathrm{c}}=-0.75 \mathrm{~V}$ : (a) $t_{\mathrm{c}}=40 \mathrm{~s}$ and (b) $t_{\mathrm{c}}=80 \mathrm{~s}$.

directions is much slower than that in other directions, such as the $\langle-110\rangle$ direction, as seen in Fig. 2b. Finally, the surface area of $\{001\}$ planes occupies a large part of the wall surface, and this results in the slow etching rate of the nanowalls. This model qualitatively explains the results obtained here. However, another possibility related to $\mathrm{H}_{2}$ evolution is not negligible. By applying the cathodic bias in the acid electrolyte, the $\mathrm{H}_{2}$ evolution will probably be induced as follows

$$
2 \mathrm{H}^{+}+2 \mathrm{e}^{-} \rightarrow \mathrm{H}_{2}
$$

This reaction takes place in parallel with the cathodic decomposition reaction described in Eq. 1. When the $\mathrm{H}_{2}$ evolution becomes dominant in the cathodic reactions, the InP walls will not dissolve any further using the competitive decomposition process. Further inves-

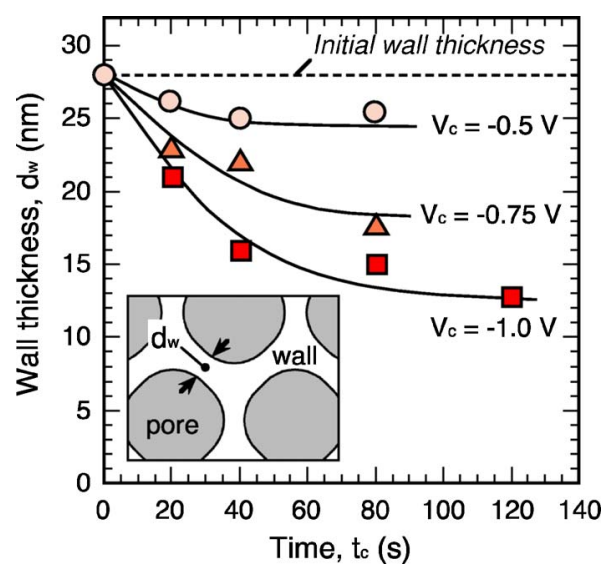

Figure 3. (Color online) Relationship between average pore thickness, $d_{\mathrm{w}}$, and cathodic processing time, $t_{\mathrm{c}}$. Solid lines indicate eye-guides. 


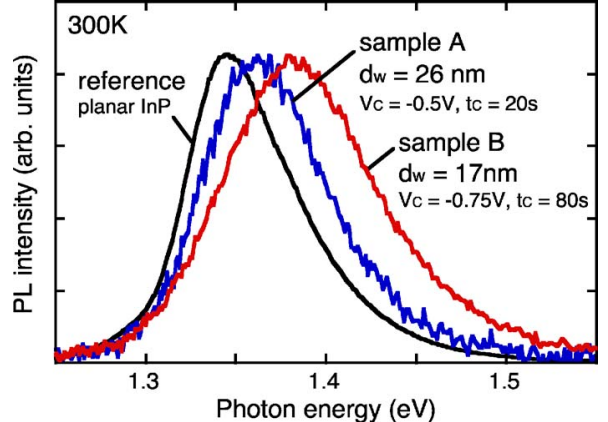

Figure 4. (Color online) PL spectra obtained from porous samples after cathodic decomposition at $V_{\mathrm{c}}=-0.5 \mathrm{~V}$ for $20 \mathrm{~s}$ (sample A) and $V_{\mathrm{c}}=-0.75 \mathrm{~V}$ for $80 \mathrm{~s}$ (sample B).

tigation is necessary for the quantitative discussion on the etching mechanism of InP nanowalls.

In order to investigate the optical properties of $\mathrm{InP}$ nanoporous structures, PL measurements were carried out on the samples with a different wall thickness after the cathodic decomposition. Figure 4 shows the result of PL measurements on the samples with a wall thickness of 17 and $26 \mathrm{~nm}$. The intensities of PL spectra are normalized by that of a planar InP substrate used as a reference. It is clearly shown that the peak positions systematically shifted to higher energy positions from that of the reference band-edge emission at $1.34 \mathrm{eV}$. As the wall thickness reduced from 26 to $17 \mathrm{~nm}$, the blue-shift values increased from 14 to $35 \mathrm{meV}$. This behavior can be explained in terms of a quantum-size effect in the InP nanowalls, as similar to the template porous samples. ${ }^{13}$ These results indicate that the cathodic decomposition process is very effective for the size tuning of InP nanoporous structures.

In summary, a two-step electrochemical process using anodic and cathodic reactions was developed to form InP-based nanostructures. The cathodic decomposition process was applied to InP porous structures for the first time, and wall thickness was precisely controlled in the range of $10-30 \mathrm{~nm}$ using cathodic conditions. The results obtained are promising for forming high-density arrays of unique nanostructures that are not obtainable using conventional methods.

\section{Acknowledgment}

This work was supported in part by the Iketani Science and Technology Foundation and the Murata Science Foundation, Japan.

Hokkaido University assisted in meeting the publication costs of this article.

\section{References}

1. Y. Arita, J. Cryst. Growth, 45, 383 (1978).

2. R. L. Smith, S.-F. Chuang, and S. D. Collins, J. Electron. Mater, 17, 533 (1988).

3. L. T. Canham, Appl. Phys. Lett., 57, 1046 (1990).

4. H. Föll, S. Langa, J. Carstensen, M. Christophersen, and I. M. Tiginyanu, Adv. Mater. (Weinheim, Ger.), 15, 183 (2003).

5. P. Schmuki, D. J. Lockwood, J. Labbe, and J. W. Fraser, Appl. Phys. Lett., 69 , 1620 (1996).

6. A. Anedda, A. Serpi, V. A. Karavanskii, I. M. Tiginyanu, and V. M. Ichizli, Appl. Phys. Lett., 67, 3316 (1995).

7. K. Kuriyama, K. Ushiyama, K. Ohbora, Y. Miyamoto, and S. Takeda, Phys. Rev. B, 58, 1103 (1998).

8. T. Takizawa, S. Arai, and M. Nakahara, Jpn. J. Appl. Phys., Part 2, 33, L643 (1994).

9. A. Hamamatsu, C. Kaneshiro, H. Fujikura, and H. Hasegawa, J. Electroanal Chem., 473, 223 (1999)

10. T. Baba and M. Koma, Jpn. J. Appl. Phys., Part 1, 34, 1405 (1995).

11. M. Mynbaeva, A. Titkov, A. Kryganovskii, V. Ratnikov, K. Mynbaev, H. Huhtinen, R. Laiho, and V. Dmitriev, Appl. Phys. Lett., 76, 1113 (2000).

12. A. P. Vajpeyi, S. Tripathy, S. J. Chua, and E. A. Fitzgerald, Physica E (Amsterdam), 28, 141 (2005).

13. H. Fujikura, A. Liu, A. Hamamatsu, T. Sato, and H. Hasegawa, Jpn. J. Appl. Phys., Part 1, 39, 4616 (2000)

14. T. Hirano, A. Ito, T. Sato, F. Ishikawa, and H. Hasegawa, Jpn. J. Appl. Phys., Part 1, 41, 977 (2002).

15. T. Sato, T. Fujino, and H. Hasegawa, Appl. Surf. Sci., 252, 5457 (2006).

16. H. Masuda, M. Ohya, K. Nishio, H. Asoh, M. Nakao, M. Nohtomi, A. Yokoo, and T. Tamamura, Jpn. J. Appl. Phys., Part 2, 39, L1039 (2000).

17. A. Gagnaire, J. Joseph, A. Etcheberry, and J. Gautron, J. Electrochem. Soc., 132 1655 (1985).

18. H. Yao and K. Itaya, J. Electrochem. Soc., 145, 3090 (1998).

19. M. Seo, M. Aihara, and A. W. Hassel, J. Electrochem. Soc., 148, B400 (2001).

20. S. Adachi and H. Kawaguchi, J. Electrochem. Soc., 128, 1342 (1981). 